\title{
M6-C artificial disc placement
}

\author{
Domagoj Coric, MD, ${ }^{1}$ John Parish, MD, ${ }^{2}$ and Margaret O. Boltes, $\mathrm{RN}^{1}$
}

${ }^{1}$ Carolina Neurosurgery and Spine Associates; and ${ }^{2}$ Carolinas Medical Center, Department of Neurosurgery, Charlotte, North Carolina

There has been a steady evolution of cervical total disc replacement (TDR) devices over the last decade resulting in surgical technique that closely mimics anterior cervical discectomy and fusion as well as disc design that emphasizes quality of motion. The M6-C TDR device is a modern-generation artificial disc composed of titanium endplates with trikeel fixation as well as a polyethylene weave with a polyurethane core. Although not yet approved by the FDA, M6-C has finished a pilot and pivotal US Investigational Device Exemption (IDE) study. The authors present the surgical technique for implantation of a 2-level M6-C cervical TDR device.

The video can be found here: https://youtu.be/rFEAqINLRCo.

KEYWORDS total disc replacement; cervical spine; artificial disc; M6-C; video 